$(\mathrm{n}=58)$. An additional 18 items were discussed and modified prior to inclusion in the Delphi.

Several themes were identified following the reflective exercise encapsulating the group experience. Motivation to work in the RSG whether professionally or personally driven felt complementary, resulting in a sense of connexion; personal/professional belonging and learning together (Table 1).

Discussion We advocate the RSG model as feasible, sustainable and enriching component of the research experience, specifically in PRO development to enhance content validity. Participating in group reflection has enhanced our understanding of the RSG dynamic.

\section{P5 QUALITY OF LIFE AND FUNCTIONAL OUTCOMES IN POST-TRANSPLANT IPF PATIENTS AGED OVER 70}

P Riddell, S Winward, K Redmond, JJ Egan. Mater Misericordiae University Hospital, Dublin, Ireland

\subsection{6/thoraxjnl-2015-207770.142}

Introduction In recent years there has been a large increase in rates of lung transplantation for IPF patients. This has driven by the introduction of the Lung Allocation Score in the US, which prioritises patients based on treatment need and benefit. Increasing rates of transplantation have led to older patients being considered for transplant listing. The aim of this study was to assess the survival, functional capacity and quality of life of IPF patients aged over 70 attending our transplant programme.

Methods Post-transplant IPF patients aged 70 years or older were identified from the National Lung Transplant Registry. Health-related Quality of Life (HRQL) was assessed using the 36-item Medical Outcomes Survey Short Form (SF-36). Functional status was assessed by exercise tolerance, pulmonary function and level of respiratory support. HRQL was compared to published datasets from randomised clinical trials of drug therapy as well as prospective studies in lung transplant recipients.

Results 6 patients met the inclusion criteria, mean age $72.5 \pm$ 0.8 yrs. The mean time from transplant was $3.8 \pm 1.5$ yrs (range 2.3 - 7.0 yrs). Compared to the BUILD-1 trial (similar age, limited IPF), minimal important clinical differences (MID) ${ }^{1}$ were seen across many components of the SF-36 score. These MIDs included physical functioning $(+7.1)$, health perception $(+29.2)$ and vitality (+17). Compared to a post-transplant cohort of younger IPF patients $(61.0 \pm 1.5 \mathrm{yrs})^{2}$ the mental component score (MCS) was higher in this study $(+12.2)$. These benefits in MCS were maintained when compared to patients in the IFIGENIA study of N-acetylcysteine and the STEP-IPF study of sildenafil. The mean reported exercise tolerance of our patient group was $1.2 \mathrm{~km}$, and no patient required supplementary oxygen or respiratory support. Compared to pre-transplant status large benefits in function were noted (mean pre-transplant 6MWT was $314 \pm 91 \mathrm{~m}$ with $6 \mathrm{~L}$ oxygen/minute).

Conclusion Lung transplantation provides clinically meaningful benefits in HRQL and functional outcomes in patient's $\geq 70 \mathrm{yrs}$ old. This study highlights that these benefits are comparable to younger IPF patients who receive lung transplant and more beneficial to those reported in drug trials.

\section{REFERENCES}

1 Respir Med. 2010;104(2):296-304

2 J Heart Lung Transplant. 2014;33(5):514-520
P6 EARLY CLINICAL EXPERIENCE WITH NINTEDANIB A TWO CENTRE REVIEW

${ }^{1} \mathrm{E}$ Nuttall, ${ }^{2} \mathrm{M}$ Crooks, ${ }^{1} \mathrm{~S}$ Gudur, ${ }^{1} \mathrm{C}$ Leonard, ${ }^{2} \mathrm{C}$ Major, ${ }^{2} \mathrm{~S}$ Hart, ${ }^{1} \mathrm{~N}$ Chaudhuri. ${ }^{1}$ University Hospital South Manchester, Manchester, UK; ${ }^{2}$ Hull and East Yorkshire Hospital NHS Trust, Hull, UK

\subsection{6/thoraxjnl-2015-207770.143}

Introduction Clinical trials of pirfenidone and nintedanib have shown similar reduced rates of lung function decline in patients with idiopathic pulmonary fibrosis (IPF). In 2013 NICE approved pirfenidone for use in IPF patients with forced vital capacity (FVC) between $50 \%$ and $80 \%$ predicted. More recently, nintedanib has been available on an individual patient supply program (IPSP).

Aims Reporting early experience of nintedanib in two tertiary referral centres, focussing on characterising the treated population, assessing the indications for use, and evaluating adverse effects.

Method All IPF patients attending two tertiary referral centre ILD clinics who were commenced on nintedanib as part of the IPSP were included. Data were collected retrospectively from clinical records and local clinical databases. Data are presented as mean (range).

Results 75 patients (mean age 70.8 years $(50-85), 76 \%$ male). The FVC was $79.2 \%$ predicted $(35 \%-123 \%)$ and transfer factor (DLCO) $45.8 \%$ predicted $(13 \%-74 \%)$ prior to commencing treatment. $54 \%$ of patients were prescribed nintedanib because they did not meet FVC criteria for pirfenidone (FVC $>80 \%$ in $41 \%$ of patients and $\mathrm{FVC}<50 \%$ in $13 \%$ ). Other indications included refusal of pirfenidone due to the side effect profile $(15 \%)$ or adverse effects requiring pirfenidone discontinuation (13\%). 39 patients (52\%) experienced adverse effects on nintedanib, the most common being diarrhoea (25\%), nausea (13\%), abnormal liver function tests (8\%) and lethargy (11\%). Adverse effects required nintedanib to be discontinued in 7 (9\%) patients (diarrhoea $(\mathrm{n}=3)$, abnormal LFTs $(\mathrm{n}=2)$ and patient choice $(\mathrm{n}$ $=2)$ ), dose reduction in $13(17 \%)$ patients, and temporarily stopped and restarted in $9(12 \%)$ patients.

Conclusion Nintedanib is a relatively new medication and although there are modest numbers in this review only $9 \%$ had to discontinue treatment. Diarrhoea is the most quoted side effect from trial data (63\% of patients in INPULSIS-2), but in our observational data only one quarter suffered diarrhoea and only 3 patients stopping due to this. Although the data is from early experience the discontinuation rate is favourable compared with published and local data on pirfenidone (drop out rate $15 \%)$. This needs continued review to further evaluate drug tolerability and real world efficacy.

\section{\begin{tabular}{l|l} 
P7 & INTERIM ANALYSIS OF NINTEDANIB IN AN OPEN-LABEL
\end{tabular}} EXTENSION OF THE INPULSIS ${ }^{\circledR}$ TRIALS (INPULSIS $^{\circledR}$-ON)

${ }^{1} \mathrm{~B}$ Crestani, ${ }^{2} \mathrm{~T}$ Ogura, ${ }^{3} \mathrm{~K}$ Pelling, ${ }^{4} \mathrm{C}$ Coeck, ${ }^{5} \mathrm{M}$ Quaresma, ${ }^{6} \mathrm{M}$ Kreuter, ${ }^{7} \mathrm{M}$ Kaye. ${ }^{1}$ Hôpital Bichat, Pneumologie, Paris, France; ${ }^{2}$ Department of Respiratory Medicine, Kanagawa Cardiovascular and Respiratory Center, Yokohama, Kanagawa, Japan; ${ }^{3}$ Boehringer Ingelheim Ltd, Bracknell, UK; ${ }^{4}$ SCS Boehringer Ingelheim Comm. V., Brussels, Belgium; ${ }^{5}$ Boehringer Ingelheim Pharma GmbH \& Co. KG, Ingelheim Am Rhein, Germany; ${ }^{6}$ Department of Pneumology, Thoraxklinik, University of Heidelberg, and Translational Lung Research Center Heidelberg, German Center for Lung Research, Germany; ${ }^{7}$ Minnesota Lung Center, Ltd., Minneapolis, Minnesota, USA

10.1136/thoraxjnl-2015-207770.144 